\title{
Quality and Customer Satisfaction Health Accessibility Framework using Social Media Platform
}

\author{
Abdulaziz S. Albarrak \\ Claremont Graduate University \\ Abdulaziz.Albarrak@,cgu.edu
}

\author{
Yan Li \\ Claremont Graduate University \\ $\underline{\text { Yan.Li@.cgu.edu }}$
}

\begin{abstract}
Access to healthcare refers to the ability of individuals to obtain needed healthcare services. It is a complex and multidimensional phenomenon, and can be affected by multiple factors. Among these factors are quality and patient satisfaction. In this study, we propose a framework, namely, Quality and Customer Satisfaction Health Accessibility Framework (QCSHAF), that takes into consideration quality and customer satisfaction in measuring health accessibility. The proposed framework utilizes different social media platforms to derive measures for quality and customer satisfaction of a health facility or physician. The framework is evaluated using a case study in three counties in Southern California. The result from the QCSHAF is compared with the E2SFCA method, a most used method in healthcare accessibility. We discuss the similarity and variation in the accessibility index values between the two methods and highlight the theoretical and practical contributions of the study.
\end{abstract}

\section{Introduction}

Health access is a fundamental goal of health care. Ensuring equitable and adequate access for the entire population is vital in order to gain and maintain a healthy life. Although health access is generally referred to as the ability of a population to gain the health services they need, it is a more complex concept that involves multidimensional factors [1, 2, 3, 4]. According to Penchansky and Thomas [1], access is defined as the degree of fit between people and healthcare system characteristics presented in five dimensions: availability, accessibility, accommodation, affordability and acceptability. They have identified that access dimensions could influence access in three ways: the utilization of health services, consumer satisfaction with the service they received, and physician work practice. Aday and Andersen [2] proposed a health access framework covering five factors that influence access: health policy, characteristics of the population, characteristics of the health system, the utilization of health services, and customer satisfaction. Although quality and customer satisfaction have been identified as critical factors to ensure better access and health care, they have yet to be fully utilized in the health accessibility measure.

Recent initiatives from the Centers for Medicare and Medicaid Services (CMS) have ensured health facilities deliver some quality measures [5]. It could penalize up to $2 \%$ from the payment to the health facility for not reporting these measures. For example, Consumer Assessment of Healthcare Providers and Systems (CAHPS) survey is one of the measures that evaluate the customer experience and satisfaction of the services they have received [36]. With the evolution of social media platforms, people have become smarter as to where they obtain the health services they need. More and more people would check reviews at Yelp.com or healthgrades.com before deciding which doctor or health facility they would select. A study conducted in Michigan found $65 \%$ of people in the U.S review the rating of physicians [6]. Another study found that almost $50 \%$ of the surveyed customers would review online ratings, and $67 \%$ of those individuals have utilized the reviews in making their decisions [7]. The UNICEF and World Health Organization have expressed that accessibility should not be measured by ratio between population and number of service providers [8]. Instead, people should receive the health care they need and by the methods and approaches that satisfy them. This study seeks to address this research gap by integrating healthcare service quality, more specifically customer satisfaction, within the existing health accessibility measures.

Health access has traditionally been measured by the availability of health care providers (supply) and the population living in the area (demand). The balance between the two factors is not usually equally distributed. The U.S government has two programs, health professional shortage areas (HPSAs) and 
medically underserved areas (MUAs) or populations (MUPs), to help government officials and policy makers identify areas where health care services are needed the most [11]. Researchers have been proposing new or improved spatial health accessibility measures to identify the level of health access available for different areas. A popular family of methods was first proposed by Radke and $\mathrm{Mu}$ called Floating Catchment Area (FCA) Methods and has been used widely to measure health accessibility $[12,13,14,15]$. Although the method family has gone through many improvements and enhancements, no existing studies include the quality of services in health access measures. This study will build upon the existing family of FCA methods to integrate quality of health in terms of customer satisfaction.

In this study, we propose a framework that integrates service quality and customer satisfaction within existing health accessibility indexes. More specifically, the service quality and customer satisfaction will be measured using data extracted from social media platforms. For example, Yelp is a leading social media platform that enables people to rate and write reviews on businesses, including health care facilities. Some studies have been conducted using Yelp to investigate patient experience and satisfaction using Yelp ratings and reviews [33, 34].

The rest of paper is presented as follows. In section 2, we review existing health accessibility literature. In section 3, we present the proposed framework. In section 4, we propose an improvement of customer satisfaction weight measures using text analysis. In section 5, we describe the research methodology and the data collection procedure. We then present the results of our methods in section 6 and framework evaluation in section 7 . We discuss the evaluation results in section 8 , and present our conclusions and limitations in the last section.

\section{Literature Review}

\subsection{Health Access Framework}

According to Aday and Andersen, access is "... actual use of personal health services and everything that facilitates or impedes their use" [2]. They proposed a healthcare utilization model that contains five factors influencing access, which include health policy, characteristics of the population, characteristics of the health system, the utilization of health services, and customer satisfaction [2]. These components can directly and indirectly affect each other. For example, health care delivery can directly affect populations at risk by accepting more low cost insurance. Also, health care delivery can directly affect the utilization of the service and customer satisfaction and indirectly impact characteristic of populations at risk. The utilization of the service and customer satisfaction can also affect other factors [4]. Following the introduction of the model in 1974, the utilization model has evolved [3, 4] from initially intended focus on family to individuals as unit of analysis. The current model [4] includes four main components: contextual characteristics, individual characteristics, health behavior and health outcome. Contextual and individual characteristics are further categorized into three elements: predisposing, enabling, and need. Predisposing is the characteristic of the population such as sex, race and values. Enabling refers to the means available to the population including both individual means (income, insurance) and community means (rural, region). Need refers to the illness of the population. Customer satisfaction involves factors that influence the outcome of the service provided to the person who seeks the healthcare. All these elements are interconnected and can provide feedback to each other in the improvement of health access.

Penchansky and Thomas have defined access as “... a general concept that summarizes a set of more specific dimensions describing the fit between the patient and the health care" [1]. These dimensions include availability, accessibility, accommodation, affordability and acceptability. Acceptability refers to the patient's attitude and acceptance of the health provider. They also indicated that these dimensions can influence the customer satisfaction, which may in turn, influence the utilization of health services. Similarly, Peters et al [9] have integrated a conceptual framework for assessing access to health services. The framework includes four dimensions; geographic accessibility, availability, acceptability and financial accessibility. The acceptability refers to patients' expectations of the quality of service and the characteristics of the health facility available. Although acceptability, satisfaction and quality of health are identified in the literature, there is still lack of research in integrating it into the health accessibility methods. In this study, we address this gap by proposing a framework that integrates satisfaction and quality of health within the health acceptability.

\subsection{Spatial Health Accessibility Methods}

Spatial health accessibility is a fundamental part of health access. Many studies have been conducted to measure the spatial health accessibility $[12,13,14,15$, 16]. Among these studies, the Two-Step Floating Catchment Areas (FCA) method is a well-recognized approach. It was first introduced by Radke and $\mathrm{Mu}$ 
[12] and then improved by Luo and Wang [13]. It is called two-step because it computes the health accessibility index in two steps. First, it calculates the physician to population ratio $(\mathrm{Rj})$ within specific travel time catchment area for each physician. Second, it sums the total physician to population ratio $(\mathrm{Rj})$ that falls within a 30-minute travel time from each population location.

Since the introduction of $2 \mathrm{SFCA}$, researchers have proposed various improvement and enhancement to the method. Most of these improvements intend to improve the distance decay function in 2SFCA. Luo and Qi [14] proposed an enhanced two-step floating catchment area (E2SFCA) that divides the travel time catchment into 3 sub-catchments (0-10, 10-20, and 2030 minutes) to address the variation of travel time within each catchment [14]. McGrail and Humphreys found [15] that metropolitan areas should have a lower catchment size than rural areas. Assuming that the availability of other nearby facilities influences the population demand of a health care provider, Wang et al. proposed a three-step floating catchment area (3SFCA) [17] method based on E2SFCA. Langford et al. [18] proposed another enhancement to 2SFCA by addressing the difference in travel time between different transportation modes such as public buses or private car [18].

Other improvements have attempted to integrate the non-spatial factors in the health accessibility method [25, 26, 27]. A recent study conducted by Li et al. [27] proposed a framework to integrate spatial and nonspatial health accessibility factors by assigning weights for each non-spatial factor based on its importance. Wang and Luo [26] have also studied integrating spatial and non-spatial factors within 2SFCA using principle component analysis. However, these existing spatial accessibility studies have not investigated how to integrate the customer satisfaction and quality of healthcare services, a vital factor for measuring healthcare access.

\subsection{Quality and Customer Satisfaction in Health Care Access}

Quality of health care is a vital component of healthcare access. As mentioned earlier, the healthcare utilization model [4] includes the customer satisfaction as an important factor that influences health access. Customer satisfaction refers to the perception of people to the quality of health services provided to them. These perceptions are related to many aspects of health access, such as convenience of care, cost of services, waiting time in physicians' offices, availability, easiness to get an appointment, the courtesy of care given by health providers, the communication between patient and physician, the patient's perception of the quality provided, etc. According to Penchansky and Thomas [1], provider characteristics could influence the patient's satisfaction, which may further impact the utilization of the healthcare services. According to Andersen and Davidson [4], "Consumer satisfaction is how individuals feel about the healthcare they receive. It can be judged by patient ratings of waiting time, travel time, communication with providers, and technical care received. From a health plan perspective, an ultimate outcome measure of patient satisfaction in this era of managed care might be whether or not enrollees choose to switch plans" [4]. In addition, Ware et al. [10] identified eight dimensions related to patient satisfaction. They are: interpersonal manner, technical accuracy, accessibility or convenience, availability, finance, efficiency, continuity, and physician environment. The multi-dimensionality of health quality and customer satisfaction can have a different effect on people's health access.

The Medicare and Medicaid Services (CMS) proposed programs and initiatives to ensure people get quality of care [5]. These programs include Physician Quality Reporting System (PQRS), Electronic Health Records Incentive Program (HER), Value-based Payment Modifier (Value Modifier), Consumer Assessment of Healthcare Providers and Systems (CAHPS), among others. Within these programs, CAHPS is a set of surveys designed to collect standardized information from patients about their experience with the healthcare providers and plans [36]. It intends to not only help consumers and patients to make better decisions regarding their healthcare access, but also help healthcare providers to identify areas for improving customer experience by comparing their CAHPS scores with benchmarks. The CMS penalizes up to $2 \%$ from the payment to healthcare providers for not providing the quality measures imposed by CMS. However, social media platforms have not been utilized in these existing measures and programs, although many studies have used Yelp reviews on patient experiences and satisfaction [33, 34].

\section{The Quality and Customer Satisfaction Health Accessibility Framework}

This study intends to propose Quality and Customer Satisfaction Health Accessibility Framework (QCSHAF) that incorporates consumer satisfaction of the healthcare providers within existing healthcare accessibility methods. The framework assumes that the customer satisfaction and the quality of the service influence people access to health care as indicated by 
Aday and Andersen [2]. The framework consists of four steps as shown in the Figure 1. In the following section, we describe each step in detail.

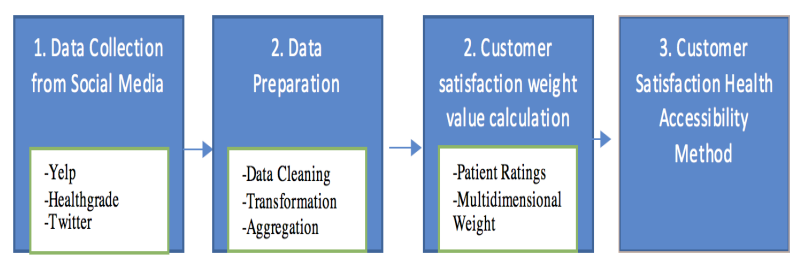

Figure 1. Quality and Customer Satisfaction Health Accessibility Framework (QCSHAF)

\subsection{Data Collection from Social Media}

Social media platforms provide a rich amount of data related to people satisfaction and experiences with healthcare service providers. This step aims to collect perceived healthcare quality and patient satisfaction data from different social media platforms, such as Yelp, Healthgrade, twitter, and others. Many social media platforms, such as Yelp, provide an API that enables developers and researchers to gather information related to users and businesses. These APIs can be used to gather the relevant data.

\subsection{Data Preparation}

This step intends to prepare the data gathered from step 1 for analysis in step 3. This involves data cleaning, such as deleting duplicates, removing or replacing missing values, etc. It may also involve aggregating or transforming variables. For example, a provider may be rated in a 5-point scale, and the satisfaction rating may be transformed into a 2-level one (i.e., satisfactory or unsatisfactory).

\subsection{Customer Satisfaction Weight Value Calculation}

This step intends to assign weights for the customer satisfaction scores. We assume that physicians that have lower consumer satisfaction scores would be less accessible than physicians with better scores in the same area, since people would usually seek the care from the later. The weight of customer satisfaction scores can be calculated in different ways. A simplest way is to directly use the healthcare provider's rating given from people. A more complex way can cover the multidimensional aspects of patient customer satisfaction as discussed in section 4 .

\subsection{Customer Satisfaction Health Accessibility Method}

The Customer Satisfaction Health Accessibility Method is an extension of E2SFCA, one of the Float Catchment Areas (FCA) family methods [14]. It integrates consumer satisfaction within the E2SFCA. The proposed method includes two sequential steps. The first step is to calculate the physician to population ratio for each facility $R_{j}$ by generating a catchment area for each physician $\mathrm{j}$. The catchment area is determined by a 30-minute travel time between physician $\mathrm{j}$ and population $\mathrm{k}$. The catchment is divided into three zone 0-10,10-20 and 20-30 minutes to accommodate the travel time difference within each catchment. The first step is computed using Eq (1),

$R_{j}=\frac{S_{j} Q_{j}}{\sum_{k \in\left(\mathrm{d}_{\mathrm{kj}} \leq \mathrm{Zr}_{\mathrm{r}}\right)} \mathrm{P}_{\mathrm{k}} \mathrm{Wr}_{\mathrm{r}}}$

where $S_{j}$ is the number of physicians at location $j$; $Q_{j}$ is the weight of consumer satisfaction with the physicians which is the Yelp rating score of the physicians as indicated in section $3.3 ; \mathrm{P}_{\mathrm{k}}$ is the population whose centroid falls within the catchment $\mathrm{k} ; \mathrm{W}_{\mathrm{k}}$ is the distance decay weight for the each zones $(1,0.42$, and 0.09 respectively) adopted from Luo and Qi [14]; $\mathrm{d}_{\mathrm{kj}}$ is the travel time between physician and population; $Z_{\mathrm{r}}$ is the travel distance zone $(1=0-10,2=10-20$ and $3=20-$ 30 minutes) within $\mathrm{k}$. The second step calculates the overall accessibility of each census track by generating a catchment area for each population $j$ and the summing up the physician to population ratio generated from step 1 that falls within a 30-minute travel time from the centroid of population $\mathrm{j}$. The second step is computed using Eq (2),

$A i c=\sum_{j \in\left(\mathrm{d}_{\mathrm{ij}} \leq \mathrm{d}_{0}\right)} R_{j} W_{j}=\sum_{j \in\left(\mathrm{d}_{\mathrm{kj}} \leq \mathrm{d} 0\right)} \frac{S_{j} Q_{j}}{\sum_{k \in\left(\mathrm{d}_{\mathrm{k} j} \leq \mathrm{Z}_{\mathrm{r}}\right)} \mathrm{P}_{\mathrm{k}} \mathrm{W}_{\mathrm{r}}}$

where $A_{i c}$ represents the accessibility at location $i$ to physicians; $R_{j}$ is the physician-to-population ratio at $j$ whose centroid falls within the catchment of $i$; and $d_{i j}$ is the travel time between $i$ and $j$. Higher value of $A_{i c}$ means better accessibility for $i$.

\section{A Proposed Conceptual Improvement of Customer Satisfaction Weight Using Text Analysis}

Customer satisfaction and healthcare service quality include different dimensions, such as waiting time, communication with physicians, availability of 
physicians, cleanness, and technical care received $[2,4$, 10]. Therefore, we propose a conceptual measure for customer satisfaction to accommodate these different dimensions. We present a proof of concept development of the conceptual measure by analyzing yelp reviews using text analysis. More specifically, an unsupervised text analysis technique, latent Dirichlet allocation (LDA) and Spearman's rho, is utilized to obtain topics underlying the Yelp reviews of physician in three counties of southern California. The LDA result highlights four important topics; communication with physician, appointment waiting time, received care, and patient experience. Each topic is represented by a group of words (table 1) and is viewed as a dimension of patient satisfaction. We then use Spearman's rho correlation to measure the correlation between each topic and the Yelp rating. The correlation coefficients are then as the weights for each patient satisfaction dimension, as shown in Eq (3),

$\mathrm{Q}=0.16 \mathrm{C}+0.21 \mathrm{P}+0.25 \mathrm{R}+0.21 \mathrm{X}$

where $\mathrm{Q}$ is the service quality and patient satisfaction score; $\mathrm{C}$ is the Communication with physician; $\mathrm{P}$ is the appointment waiting time; $\mathrm{R}$ is the received care; and $\mathrm{X}$ is the patient experience.

Table 1. Topics generated from LDA

\begin{tabular}{|l|l|}
\hline Topic & Group of words \\
\hline $\begin{array}{l}\text { Communication with } \\
\text { physician }\end{array}$ & $\begin{array}{l}\text { Doctor, office, listen, } \\
\text { staff, wait, and love }\end{array}$ \\
\hline $\begin{array}{l}\text { Appointment waiting } \\
\text { time }\end{array}$ & $\begin{array}{l}\text { Time, doctor, wait, } \\
\text { appointment, call and } \\
\text { office }\end{array}$ \\
\hline Received care & $\begin{array}{l}\text { Doctor, great, care, } \\
\text { make, call and feel }\end{array}$ \\
\hline Patient experience & $\begin{array}{l}\text { Friendly, office, wait, } \\
\text { doctor, great and } \\
\text { recommend }\end{array}$ \\
\hline
\end{tabular}

\section{Research Methodology}

This study adopts the Design Science Research (DSR) methodology proposed by Hevner et al $[29,30]$. DSR is appropriate because the study intends to solve real world problems by building artifacts. The proposed artifact is a framework that integrates quality and customer satisfaction within existing health accessibility methods to identify areas with low health access. The method is evaluated by comparing it with the E2CFA, a popular accessibility method for measuring spatial healthcare access. A case study is conducted using data collected for three counties in
Southern California: Los Angeles, San Bernardino and Riverside Counties.

The study uses two main data sources: 2014 Esri Census Data [32] and Yelp.com. The 2014 US census data is used to obtain demographics and geographic boundaries for each census track for the case study regions. Yelp API [31] is used to collect provider reviews within the case study regions with more than 12,000 healthcare providers have been found in Yelp within these counties. In addition to the business names, reviews, ratings, the Yelp API also provides the geolocations of most providers. For the few physicians' geolocations were retrieved from Yelp API, we used Google Maps to get their coordinates.

The proposed framework implementation utilizes Esri ArcGIS. More specifically, we use Esri Network Analyst Extension to calculate the driving time between healthcare providers and census track centroids and vice versa. A 30-minute driving time is used which is divided into three zones: $0-10,10-20$ and 20-30 minutes. All results are displayed in map charts to identify areas with low health accessibility.

\section{Results}

Figures 2, 3 and 4 show the results from the QCSHAF instantiation in Los Angeles county, San Bernardino and Riverside counties, and all three counties combined. The results clearly show that urban areas generally have higher health accessibility than rural areas. This result confirms with many previous studies $[14,24,26,35]$. As shown in figure 4, the health accessibility in Los Angeles is much higher than San Bernardino and Riverside counties. This difference might be attributed to two possible explanations. First is the low number of physicians in San Bernardino and Riverside counties compared with Los Angeles County. Second is the higher customer ratings of health facilities in Los Angeles compared to Bernardino and Riverside counties, with average ratings of 3.7 and 3.2 respectively.

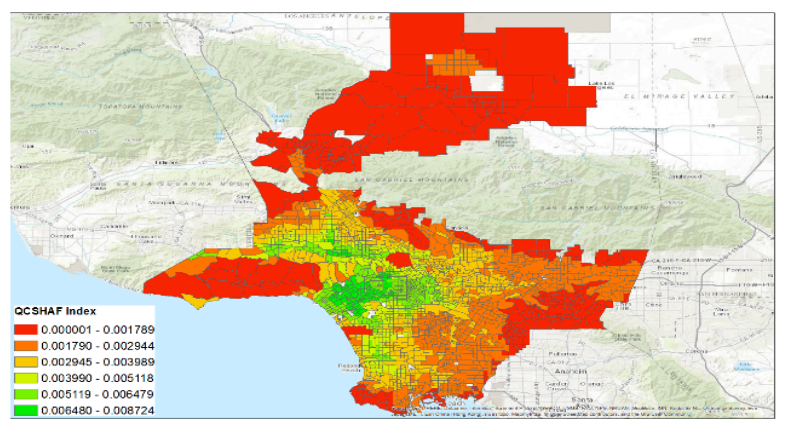

Figure 2. QCSHAF index in Los Angeles county 
The comparison between Figures 2 and 3 shows that health access is better in populated areas, especially the central parts of the cities. For example, the accessibility is lower in the eastern, southern and northern areas of Los Angeles, such as Covina, Downey, San Fernando Valley and Lancaster. This corresponds with the designated HPSA areas [11]. In San Bernardino and Riverside counties, the higher health access areas include Palm Desert and Murrieta, and lower health access areas include Redland, Highland, Banning, Hemet and Corona. Most of these low access areas are designated physician shortage areas by HPSA.

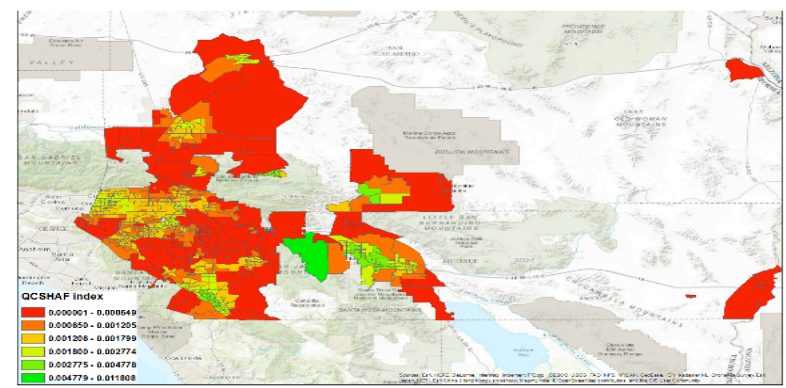

Figure 3. QCSHAF index in San Bernardino and Riverside counties

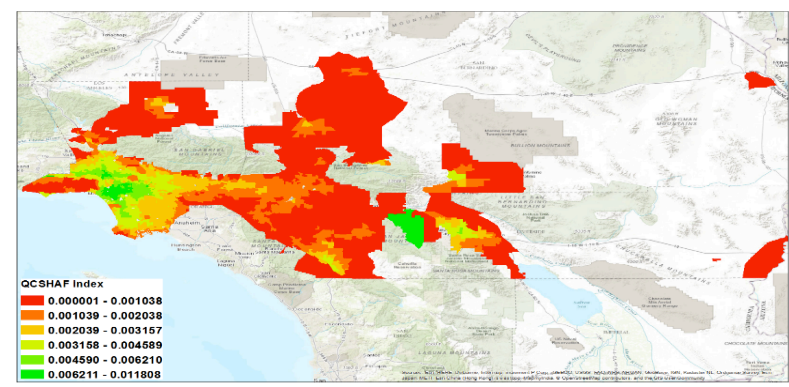

Figure 4. QCSHAF index in all three counties combined

\section{Evaluation}

The framework is evaluated by comparing its instantiation results with the E2SFCA method proposed by Luo and Qi [14] in the case study region. Figures 2, 3 and 4 shows the QCSHAF results, and Figures 5, 6 and 7 show the E2SFCA result. The comparison highlights many variations in the health accessibility index values in different areas between the two methods. For example, the QCSHAF shows different accessibility scores from the E2SFCA in the East and West areas of Los Angeles, such as Est Covina, Downey, Arcadia, El Monte and San Fernando Valley. It also shows better health access in Barstow than the E2SFCA, which may contribute to more healthcare providers with higher customer satisfactions in this area. In addition, the independent T-test shows the significant difference between the indexes values of QCSHAF and E2SFCA (i.e., $F=6025.869$, p value < $0.001, \mathrm{t}=$ 93.964). Current instantiation of the QCSHAF only includes the provider rating as the stratification score. In the future, we plan to integrate and test the proposed weighted quality scores from section 4 to accommodate different dimensions of satisfaction in the health accessibility framework.

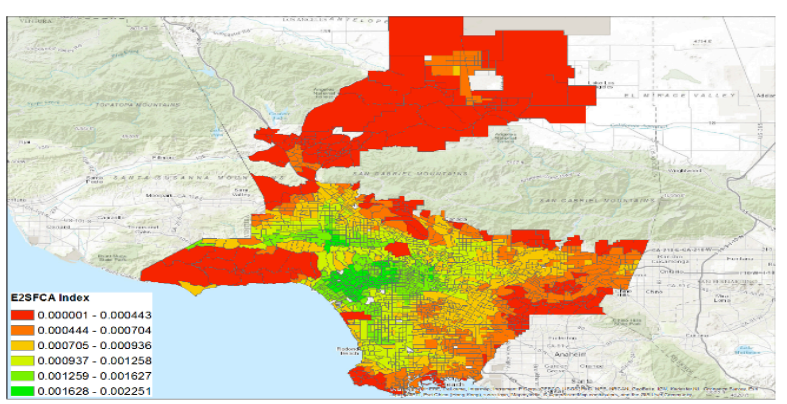

Figure 5. E2SFCA index in Los Angeles county

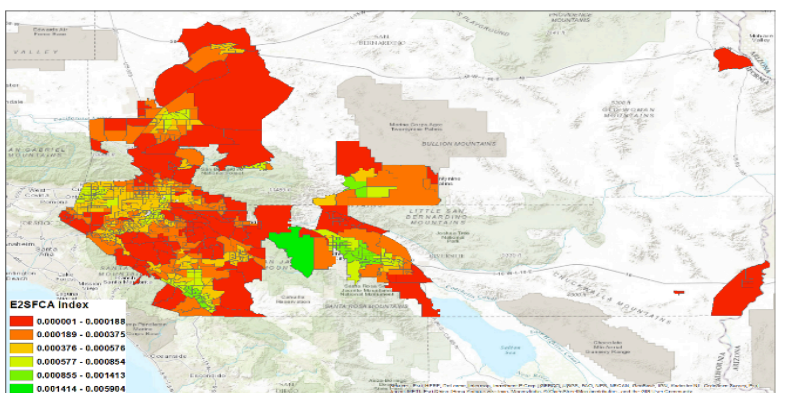

Figure 6. E2SFCA index in San Bernardino and Riverside counties

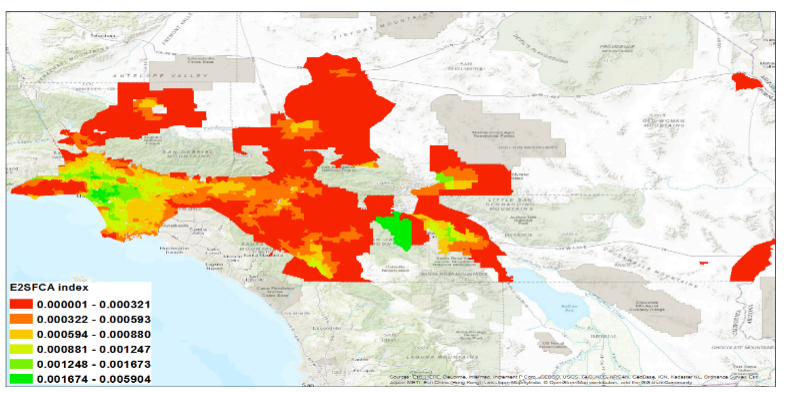

Figure 7. E2SFCA index in all three counties combined

\section{Discussions and limitations}

This study provides both theoretical and practical contributions. It proposes a novel framework that integrates quality and customer satisfaction data from 
social media platforms within the health accessibility method. It extends the floating catchment area (FCA) methods, specifically E2SFCA, by integrating customer satisfaction and service quality measures. It demonstrates that that the customer perception of the healthcare service quality from social media can be integrated with existing healthcare accessibility methods to ensure people can access the best healthcare services. Although the case study only utilizes Yelp as the social media platform, the framework can integrate data from many other social media platforms, such as healthgrade.com, twitter, etc.

This study contributes to the knowledge base of DSR by designing a novel framework, QCSHAF. The framework design utilizes multidisciplinary theories and methods to provide healthcare access measures that include quality of healthcare services. Health professionals, policy makers and insurance companies may utilize the proposed framework to identify and improve areas with low health access by allocating more resources and physicians with higher satisfactory scores.

This study is not without limitations. First, although Yelp provides a large number of physicians, it may not reflect the actual number of physicians since some physicians may not have yelp business accounts. Second, we used Yelp rating as the weight of patient customer satisfaction instead of using the proposed conceptual improvement of customer satisfaction weight (Eq3) because we do not have all the reviews for all the physicians. Future research will address this limitations by collecting reviews from multiple data sources. Third, the framework inherits some of E2SFCA method limitations, including the assignment of travel time weights [35]. Although we have adopted the sub-catchments' weights proposed by Luo and Qi [14] in this study, a better weight assignment should be based on actual utilization data.

\section{Conclusion}

Accessing healthcare service quality should be an essential aspect of the healthcare system. In this study, we are proposing the QCSHAF framework that integrates customer satisfaction and quality of healthcare services within the existing health accessibility methods. Social media platforms, such as Yelp, can be used to measure the healthcare service quality and customer satisfaction. The results from the framework instantiation show great variations in the health accessibility scores in Los Angeles, San Bernardino and Riverside counties. The framework is evaluated by comparing it with the E2SFCA method. The comparison highlights the physician ratings can be an important factor in measuring healthcare access. For future work, we plan to integrate the conceptual improvement of customer satisfaction weight within the framework as indicated in section 4 .

\section{References}

[1] Penchansky, Roy, and J. William Thomas. "The concept of access: definition and relationship to consumer satisfaction." Medical care 19.2 (1981): 127-140.

[2] Aday, Lu Ann; Andersen R (1974). "A framework for the study of access to medical care". Health Serv Res 9 (3): 20820. PMC 1071804. PMID 4436074.

[3] Andersen, Ronald (1995). "Revisiting the behavioral model and access to medical care: does it matter?". J Health Soc Behav 36 (1): 1-10. doi:10.2307/2137284. PMID 7738325

[4] Andersen, Ronald M., Pamela L. Davidson, and S. E. Baumeister. "Improving access to care in America." Changing the US health care system: key issues in health services policy and management. 3a. edición. San Francisco: Jossey-Bass (2007): 3-31.

[5] Centers for Medicare and Medicaid Services (CMS): Available http://www.cahps.ahrq.gov/cahpskit/Healthplan/HPChooseQ $\mathrm{x} 2$.asp

[6] Hanauer, David A., et al. "Public awareness, perception, and use of online physician rating sites." Jama 311.7 (2014): 734-735.

[7] PwC Scoring healthcare: Navigating customer experience ratings. [2014-03-07]. Available at: http://www.pwc.com/us/en/health-

industries/publications/scoring-patient-healthcareexperience.jhtml.

[8] UNICEF, and World Health Organization. "Primary health care: report of the International Conference on Primary Health Care, Alma-Ata, USSR, 6-12 September 1978." (1978).

[9] Peters, David H., et al. "Poverty and access to health care in developing countries." Annals of the New York Academy of Sciences 1136.1 (2008): 161-171.

[10] Ware JE, Davies-Avery A, Stewart AL. The measurement and meaning of patient satisfaction. Health and Medical Care Services Review 1978;

[11] DHHS (Department of Health and Human Services), 2000. Lists of designated primary medical care, mental health, and dental health professional shortage areas; notice. Federal Register 65 (180), 56028-56075. 
[12] Radke, J., Mu, L., 2000. Spatial decomposition, modeling and mapping service regions to predict access to social programs. Geographic Information Sciences 6, 105112 .

[13] Luo, Wei, and Fahui Wang. "Measures of spatial accessibility to health care in a GIS environment: synthesis and a case study in the Chicago region." Environment and Planning B: Planning and Design 30.6 (2003): 865-884.

[14] Luo, Wei, and Yi Qi. "An enhanced two-step floating catchment area (E2SFCA) method for measuring spatial accessibility to primary care physicians." Health \& place 15.4 (2009): 1100-1107.

[15] McGrail, Matthew R., and John S. Humphreys. "Measuring spatial accessibility to primary care in rural areas: improving the effectiveness of the two-step floating catchment area method." Applied Geography 29.4 (2009): 533-541.

[16] Luo, Wei, and Tara Whippo. "Variable catchment sizes for the two-step floating catchment area (2SFCA) method." Health \& place 18.4 (2012): 789-795.

[17] Wan, Neng, Bin Zou, and Troy Sternberg. "A three-step floating catchment area method for analyzing spatial access to health services." International Journal of Geographical Information Science 26.6 (2012): 1073-1089.

[18] Langford, Mitchel, Gary Higgs, and Richard Fry. "Multi-modal two-step floating catchment area analysis of primary health care accessibility." Health \& place 38 (2016): 70-81.

[19] Dai, Dajun, and Fahui Wang. "Geographic disparities in accessibility to food stores in southwest Mississippi." Environment and Planning B: Planning and Design 38.4 (2011): 659-677.

[20] Polzin, Pierre, José Borges, and António Coelho. "An extended kernel density two-step floating catchment area method to analyze access to health care." Environment and Planning B: Planning and Design 41.4 (2014): 717-735.

[21] Luo, Jun. "Integrating the Huff model and floating catchment area methods to analyze spatial access to healthcare services." Transactions in GIS 18.3 (2014): 436448 .

[22] Siegel, Martin, et al. "Developing a composite index of spatial accessibility across different health care sectors: A German example." Health Policy 120.2 (2016): 205-212.

[23] Delamater, Paul L. "Spatial accessibility in suboptimally configured health care systems: A modified two-step floating catchment area (M2SFCA) metric." Health \& place 24 (2013): 30-43.
[24] Mao, Liang, and Dawn Nekorchuk. "Measuring spatial accessibility to healthcare for populations with multiple transportation modes." Health \& place 24 (2013): 115-122.

[25] Wang, Fahui. "Measurement, optimization, and impact of health care accessibility: a methodological review." Annals of the Association of American Geographers 102.5 (2012): 1104-1112.

[26] Wang, Fahui, and Wei Luo. "Assessing spatial and nonspatial factors for healthcare access: towards an integrated approach to defining health professional shortage areas." Health \& place 11.2 (2005): 131-146.

[27] Li, Yan, et al. "Designing utilization-based spatial healthcare accessibility decision support systems: A case of a regional health plan." Decision Support Systems (2017).

[28] Vo, Au, Miloslava Plachkinova, and Rahul Bhaskar. "Assessing healthcare accessibility algorithms: A comprehensive investigation of two-step floating catchment methodologies family." (2015).

[29] Von Alan, R. Hevner, et al. "Design science in information systems research." MIS quarterly 28.1 (2004): 75-105.

[30] Alan Hevner \& Samir Chatterjee. Design Research in Information Systems: Theory and Practice (Integrated Series in Information Systems). Springer. Edition: 2010th.

[31] Yelp API. Retrieved September, 2017 from https://www.yelp.com/developers/documentation/v3

[32] Esri Demographics Data. Available at http://doc.arcgis.com/en/esri-demographics/data/us-intro.htm

[33] Ranard, Benjamin L., et al. "Yelp Reviews Of Hospital Care Can Supplement And Inform Traditional Surveys Of The Patient Experience Of Care." Health Affairs 35.4 (2016): 697-705.

[34] Rastegar-Mojarad, Majid, et al. "Collecting and analyzing patient experiences of health care from social media." JMIR research protocols 4.3 (2015): e78.

[35] Wang, Lu, and Travis Tormala. "Integrating spatial and aspatial factors in measuring accessibility to primary health care physicians: a case study of Aboriginal population in Sudbury, Canada." J Community Med Health Educ 4.284 (2014): 2161-0711.

[36] Agency for Healthcare Research and Quality (AHRQ). CAHPS Health Plan Survey 4.0: Adult Commercial Instrument, CAHPS $®$ Health Plan Survey and Reporting Kit 2008. Rockville (MD): AHRQ; 2008. Available at http://www.cahps.ahrq.gov/cahpskit/Healthplan/HPChooseQ $\mathrm{x} 2$.asp 